\title{
Legacy and Emerging Contaminants in Plants: From the Gene to the Field
}

\author{
Conceição Santos, ${ }^{1}$ Helena Oliveira, ${ }^{2}$ Joanna Deckert, ${ }^{3}$ and Jason C. White ${ }^{4}$ \\ ${ }^{1}$ Laboratory of Biotechnology and Cytomics, UA and CESAM, 3810-193 Aveiro, Portugal \\ ${ }^{2}$ Department of Biology, CESAM, University of Aveiro, 3810-193 Aveiro, Portugal \\ ${ }^{3}$ Department of Plant Ecophysiology, Adam Mickiewicz University, 61614 Poznań, Poland \\ ${ }^{4}$ Department of Analytical Chemistry, Connecticut Agricultural Experiment Station, 123 Huntington Street, \\ New Haven CT 06504, USA
}

Correspondence should be addressed to Conceição Santos, csantos@ua.pt

Received 26 September 2012; Accepted 26 September 2012

Copyright (c) 2012 Conceição Santos et al. This is an open access article distributed under the Creative Commons Attribution License, which permits unrestricted use, distribution, and reproduction in any medium, provided the original work is properly cited.

It has become increasingly clear over the last decades that the interdependence of economic development and environment sustainability is a crucial matter for human health and well-being and as such, far surpasses the restricted forums of scientific academia. In particular, and despite increasing legislation to restrict soil and water contamination in most countries, contamination in agricultural areas remains a growing problem, with clearly recognized negative impacts on plant and animal life. Mining, industry, overuse of pesticides and chemical fertilizers, and improper waste disposal can all contribute to soil contamination. Moreover, some of the most intense effects of soil and water contamination involve putative decreases of crop yield and/or food contamination and ultimately the complete loss of usable land (and habitats).

The papers of this special issue, discuss various facets of soil contamination - plant interactions that are of critical importance in the third millennium. The papers were thoughtfully designed as reviews covering diverse subjects of plant responses to different types of soil contaminants, thereby providing an overview of the recent trends in this field of study.

The arrangement of subjects is as follows: B. Ladeiro discusses and updates some recent aspects related to soil degradation resulting from salt stress and the opportunities of saline agriculture in these areas. The authors also include brief considerations on the valorization of halophyte crops in order to address the requirements for agriculture improvement and adjustments in the 21 st century.

Some other papers in this issue explore the traditional problem of soil contamination by metals and the resulting impacts on plant performance and agriculture. In spite of the fact that metals' adverse effects have been known for decades, exposure to this type of contaminant continues and is even increasing in several countries. In this issue authors review the toxicity of some metals, such as lead, aluminum, mercury, and chromium. Lead is a common environment pollutant, though less studied as a toxicant to plants, than other metals such as cadmium. S. Pinho and B. Ladeiro discuss lead phytotoxicity, reviewing some aspects of toxicity mechanisms in plants, focusing on metabolic pathways, in particular oxidative stress - an issue yet to be fully clarifiedand its accumulation in crops and putative perspectives of phytoremediation. S. Silva clearly describes some of the most recent advances in aluminum-induced phytotoxicity, with emphasis on aspects such as root growth inhibition, oxidative stress, alterations on cell wall and plasma membrane, nutrient unbalances, callose accumulation, and disturbance of cytoplasmic $\mathrm{Ca}^{2+}$ homeostasis.

R. Azevedo and E. Rodriguez deal with the phytotoxicity of mercury in plants, a still poorly understood pollutant. The authors cover different environment aspects of this pollutant and, in particular update most recent data on $\mathrm{Hg}$ accumulation and genotoxicity in some crops. 
H. Oliveira reviews several aspects of chromium accumulation in soil and its phytotoxicity. Evaluating the chromium toxicity profile is complex due to several valence states, which have different pathways of entrance, and act in different ways inside the cell.

Finally, two reviews deal with two classes of emerging contaminants, reviewing key aspects of nanoparticle and pesticide phytotoxicity. In the first review, C. Remédios and co-authors discuss the main topics of nanoparticle contamination and the potential toxic effects of metal-based nanoparticles in plants, which remain largely unknown. In the latter, M. C. Dias reviews and updates some crucial aspects of fungicides' (extensive) use in agriculture. The author highlights some recent effects of fungicides in plant physiology with particular emphasis on photosynthesis.

\section{Conceição Santos \\ Helena Oliveira Joanna Deckert Jason C. White}



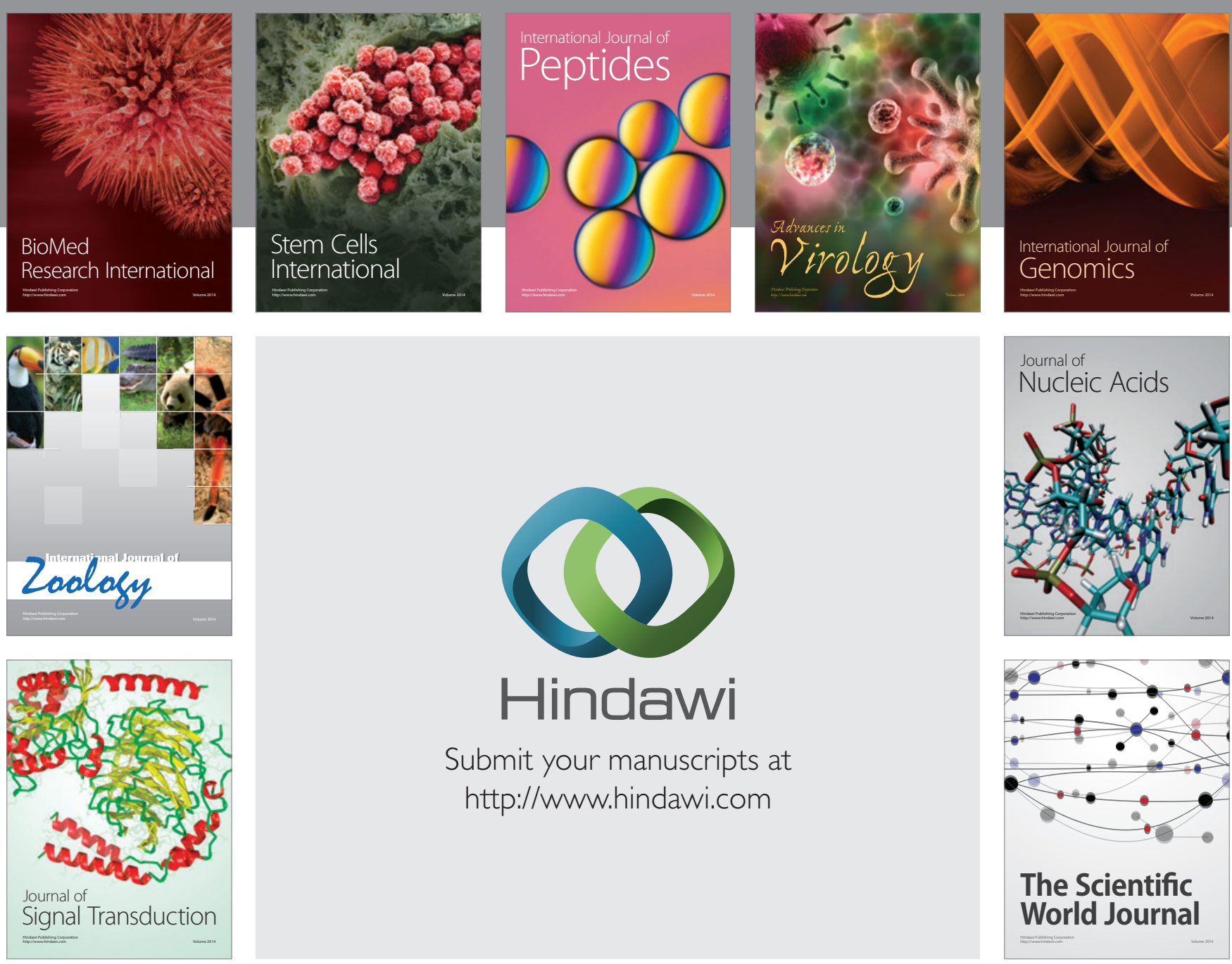

Submit your manuscripts at

http://www.hindawi.com
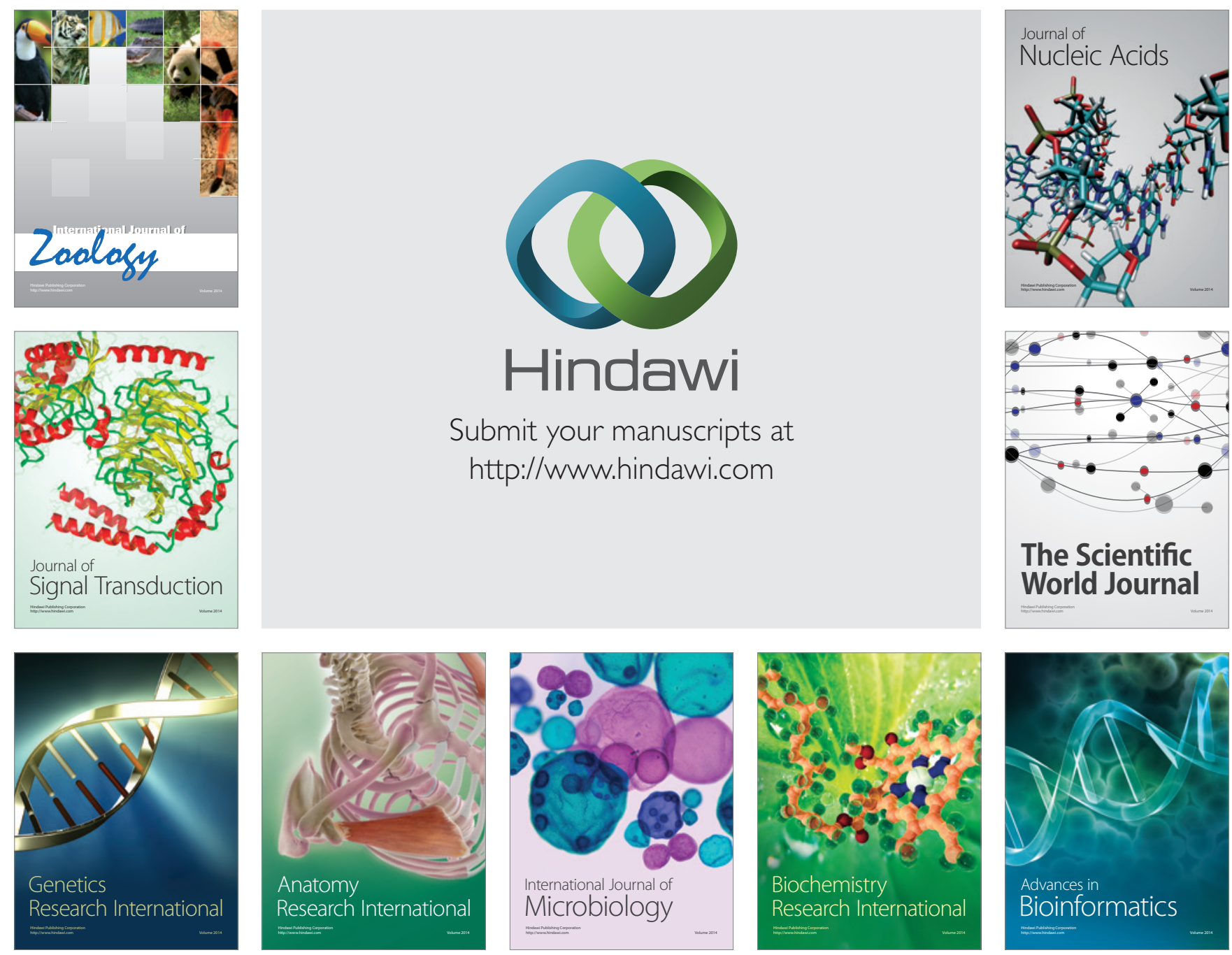

The Scientific World Journal
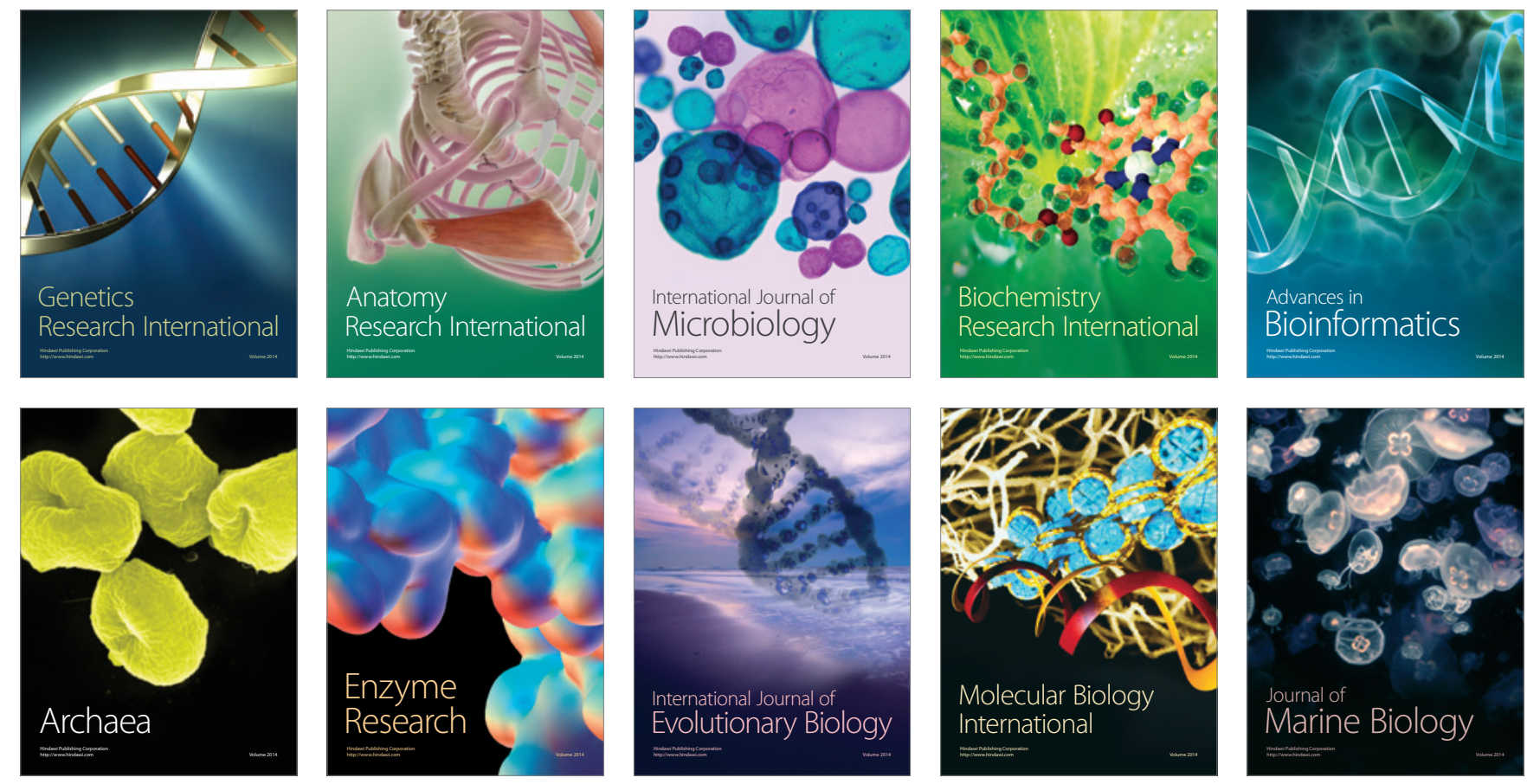